Research Article

\title{
Polarized Laser Backscattering of Atmospheric Cloud Distribution Based on Simulated Annealing Algorithm
}

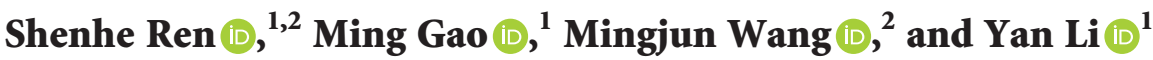 \\ ${ }^{1}$ School of Optoelectronic Engineering, Xi'an Technological University, Xian 710048, China \\ ${ }^{2}$ School of Physics and Electronic Engineering, Xianyang Normal University, Xian 710048, China \\ Correspondence should be addressed to Ming Gao; brean14na@mail.alumni.edu.vn
}

Received 25 November 2020; Revised 22 February 2021; Accepted 2 March 2021; Published 4 May 2021

Academic Editor: Fangqing Wen

Copyright (c) 2021 Shenhe Ren et al. This is an open access article distributed under the Creative Commons Attribution License, which permits unrestricted use, distribution, and reproduction in any medium, provided the original work is properly cited.

\begin{abstract}
Meteorological forecasting can not only reduce the losses caused by natural disasters to human society but also has a very important significance in the fields of water conservancy, aviation, and transportation. In order to improve the accuracy of meteorological forecasting, we should focus on the in-depth optical analysis of atmospheric cloud distribution. Compared with forward-scattered laser light, backscattered laser light can save more optical information. Therefore, this paper studies the backscattering of polarized laser light distributed in atmospheric clouds. In this study, a simulated annealing algorithm was used to invert the data of spaceborne lidar to obtain the depolarization degree and backscattering coefficient of atmospheric clouds and aerosols at different heights. Finally, based on the radar measurement example, the simulated annealing algorithm was used to analyze the atmospheric information of sunny, cloudy, and hazy weather in summer and winter, and the atmospheric depolarization and backscattering coefficients corresponding to different weather heights were obtained. The corresponding cloud layer type was judged. The research results prove the feasibility of the simulated annealing algorithm in the study of polarized laser backscattering in atmospheric cloud distribution. This study provides new ideas for radar data processing methods and provides a theoretical basis for further research in the field of meteorological forecasting.
\end{abstract}

\section{Introduction}

Lidar is a detection system that analyzes the difference between the signals emitted and received by the laser beam to determine the precise location area of the target and its movement speed. It combines the characteristics of traditional radar radio detection and advanced laser technology and has many advantages such as high resolution, strong anti-interference ability, and antiground echo (Giacomo et al.) [1]. NASA successfully launched a "CALIPSO" satellite in 2006, which contains atmospheric clouds and aerosol lidar and infrared radar, namely, orthogonally polarized clouds and aerosol lidar (CALIOP), aimed at multiangle detection of the Earth's atmospheric clouds and aerosols to obtain more accurate meteorological data (Ponomarenko et al.) [2]. When electromagnetic waves are incident from a uniform medium, surface scattering occurs at the interface between the two uniform media. The scattering signal received in the direction opposite to the incident direction is forward scattering. In remote observation systems such as radar or scattering photoelectric sensors, the scattered wave is the direction of incidence, and the scattering phenomenon observed at this time is backward scattering (Rusli et al.) [3]. Scholars in the past usually used forward-scattered light for research, but forward-scattered light is prone to loss of optical information in strong scattering media, which will have a greater impact on resolution (Tian et al.) [4]. Backscatter is easier to detect than forward-scattered light, and the probability of information loss is relatively small. Therefore, in recent years, backscatter has been widely used in atmospheric remote sensing, medical detection, and other fields.

A simulated annealing algorithm is an algorithm that simulates the annealing process of solid materials. The simulated annealing algorithm uses the Monte Carlo iterative solution strategy, which has the characteristics of less 
initial condition requirements, not easily limited to local optimal values, and can solve complex problems such as multiextreme nonconvex feasible domain problems. The calculation process of radar laser scattering is very complicated and cumbersome. In order to simplify the calculation process on the basis of ensuring the accuracy of the solution set, this paper uses a simulated annealing algorithm to perform the inversion operation of CALIOP spaceborne lidar data to obtain atmospheric cloud and aerosol depolarization and backscattering coefficient at different measurement heights. It is hoped that the research results can provide reliable technical support for meteorological prediction and contribute to the further development of meteorological science.

A large amount of data of CALIOP spaceborne lidar usually uses MATLAB software for data processing, but the use of MATLAB for data processing has the disadvantages of complicated operation and long running time. In order to explore the new method of data processing for CALIOP spaceborne lidar, this study, based on the Monte Carlo method, innovatively proposed the use of a simulated annealing algorithm for CALIOP data inversion, which provides a new method for atmospheric remote sensing meteorological monitoring based on spaceborne radar ideas.

The main content of this article is divided into four parts, of which the second part gives a brief introduction to the frontier applications of backscattering in atmospheric remote sensing, medical detection, Internet of Things, and information technology. The third part is from the introduction of CALIOP spaceborne lidar, the backscattering coefficient and depolarization coefficient, the introduction of the principle of simulated annealing algorithm, the analysis of relevant data of water vapor cloud and ice crystal cloud based on simulated annealing algorithm, etc. The distribution of atmospheric clouds was studied from the angle of polarized backscatter. The fourth part analyzes the depolarization degree and backscattering coefficient of atmospheric clouds and aerosols in different seasons and weather conditions through examples and proves the feasibility of a simulated annealing algorithm used in the inversion of spaceborne lidar data. The fifth part summarizes the results of the example analysis and points out the deficiencies of this article.

\section{Related Works}

Because of its unique directional characteristics, backscattering along the incident direction has a wide range of applications in the fields of meteorology, the Internet of Things, and medical diagnosis. In order to eliminate the necessity of channel estimation in the backscatter communication system, Wang et al. designed a data detection algorithm based on differential coding and derived two closed error rate detection thresholds and corresponding bit error rate expressions. In addition, in order to simplify the performance analysis, the lower and upper bounds of the bit error rate in high SNR areas are also studied. The simulation results prove the feasibility of theoretical research (Wang et al.) [5]. Wallis and colleagues used high-resolution electron backscatter diffraction to measure the lattice orientation gradient of olivine and used a limited number of olivine slip systems to perform a simple least-squares inverse of the geometrically necessary dislocation density play. The results show that the noise baseline of geometrically necessary dislocations is inversely proportional to the step size and is proportional to the backscatter diffraction of electrons (Wallis et al.) [6]. To investigate the effect of dipeptidyl peptidase- 4 inhibitors on the animal cardiovascular system, the Nozue $\mathrm{T}$ team analyzed the effect of sitagliptin on coronary atherosclerosis in patients with type 2 diabetes by using vascular backscatter ultrasound detection. The antiatherosclerotic effect of sitagliptin was analyzed by Nozue et al. [7]. Lyu et al. proposed to use the backscatter mode to assist the communication system for information transmission. In order to maximize the throughput of the secondary communication system, the optimal time allocation between the environmental backscattering mode and the energy collection is obtained, and a numerical solution is obtained. The numerical solution proves that the backscattering mode is more than the reference mode superiority (Lyu et al.) [8]. Lurton and colleagues analyzed the azimuth effect of $300 \mathrm{kHz}$ multibeam echo sounder in shallow water cruising and gave specific measurement strategies. In some areas of shallow water, the sonar resolution is low, and it is difficult to directly observe the existence of ripples. Due to the lack of sonar resolution, it is impossible to directly observe the strong dependence of the backscatter level from the sounding data and sonar images. The basic model of backscattering at the ripple interface explains these observations (Lurton et al.) [9].

The Haeffelin $\mathrm{M}$ team introduced an experimental device based on juxtaposed automatic lidar and ceilometer measurement, relative humidity measurement, and horizontal visibility measurement to study the hygroscopic growth of fogging nodules. By using the backscattering profile of the laser radar or the ceilometer to track the hygroscopic growth of fogging tuberculosis, the law of the hygroscopic growth of the fogging tuberculosis was obtained, and finally, a method using the attenuation backscattering measurement value was proposed. The algorithm of prefog early warning evolved over time. Experiments show that the fog warning mainly occurs 10 to 50 minutes before the fog is formed, and the height is in the range of 0 to $100 \mathrm{~m}$ from the ground (Haeffelin et al.) [10]. Gebru et al. used the kilohertz optical remote sensing system to perform in situ determination of melanization and backscattering cross sections of near-infrared and short-wave infrared. The results show that the backscattering cross section in short-wave infrared is not sensitive to melanin, and the reflection of insects in shortwave infrared is stronger than near-infrared and visible light (Gebru et al.) [11]. Kim and colleagues proposed hybrid backscatter communication for wireless communication networks to increase the transmission range and maintain the uniformity of rate distribution in a heterogeneous network environment. In a heterogeneous network where high-power base stations and dense low-power access points coexist, a maximum throughput area is set according to the user's location. The research results prove that the hybrid 
backscatter communication can expand the transmission range of the wireless communication heterogeneous network and, at the same time, achieve a uniform rate distribution (Kim et al.) [12]. The Lindsley team used advanced scatterometers to reconstruct the surface radar cross-sectional image, respectively, using the weighted AVE algorithm, scatterometer image reconstruction algorithm, and traditional grid method for data processing, and the spatial resolution, pixel mean, variance, and pixels of the data. The correlation is compared, and the effective spatial resolution of the reconstructed image of the weighted AVE algorithm and the scatterometer image reconstruction algorithm is about $15 \mathrm{~km}$ to $20 \mathrm{~km}$ (Lindsley et al.) [13]. Qian proposed a transmission model suitable for the environment backscatter system. This model uses the surrounding RF signal source to send some low-rate information to the reader and then conducts all the channel state information of the system that is unknown. Basic research on noncoherent symbol detection was performed. Simulation experiment results verify the efficiency of the theoretical model (Qian et al.) [14].

In summary, backscattering contains a lot of optical information, so backscattering has strong optical significance and has extensive research value in various fields including atmospheric remote sensing and meteorological monitoring. In this paper, in order to explore the relationship between the distribution of atmospheric clouds and the optical information contained in atmospheric clouds, the phenomenon of polarized laser backscattering of CALIOP spaceborne lidar is studied.

\section{Atmospheric Cloud Distribution Based on Simulated Annealing Algorithm}

3.1. CALIOP Lidar and Backscatter. The spaceborne lidar CALIOP mainly includes two modules: laser emission and laser reception (Wenwen et al.) [15]. The launch module contains two sets of the same expanded laser launch device and laser beam control device. A typical laser launch device is shown in Figure 1. The receiving module is an optical platform equipped with telescopes, detectors, drivers, amplifiers, and other devices. The signal received by the telescope weakens the background interference through filtered light and a narrow band. The reflected light passes through the polarization beam splitter, and the polarization directions become perpendicular to each other.

The optical path of the transmitting and receiving module is shown in Figure 2. Combined with frequency doubling technology, the transmitter can output laser pulses with wavelengths of $532 \mathrm{~nm}$ and $1064 \mathrm{~nm}$ and a width of $20 \mathrm{~ns}$, with a single pulse energy of $0.11 \mathrm{~J}$ and a beam divergence angle of $110 \mu \mathrm{rad}$. Therefore, the ground light spot is $70 \mathrm{~m}$ and the horizontal resolution is $333 \mathrm{~m}$. The backscattered signal detected by the receiving system telescope is incident on the beam splitter, and the reflected light wavelength is $532 \mathrm{~nm}$. By recording the signal intensity of polarized light in different directions, we can use backscattering to obtain atmospheric cloud information.

The primary scattering equation of lidar is shown in equation (1). Among them, $z, P(z), C, \beta_{M}, \beta_{R}, \alpha_{M}$, and $\alpha_{R}$

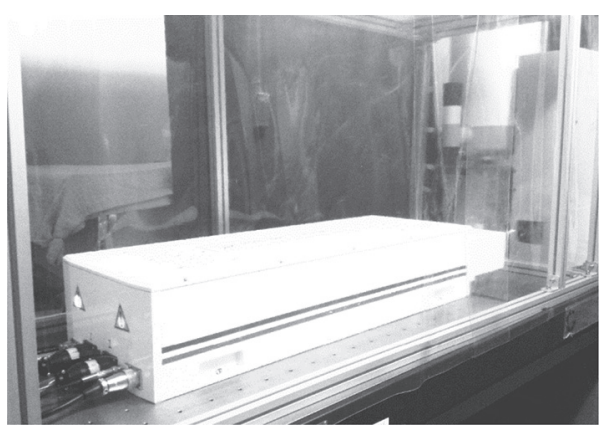

Figure 1: Typical laser emitter.

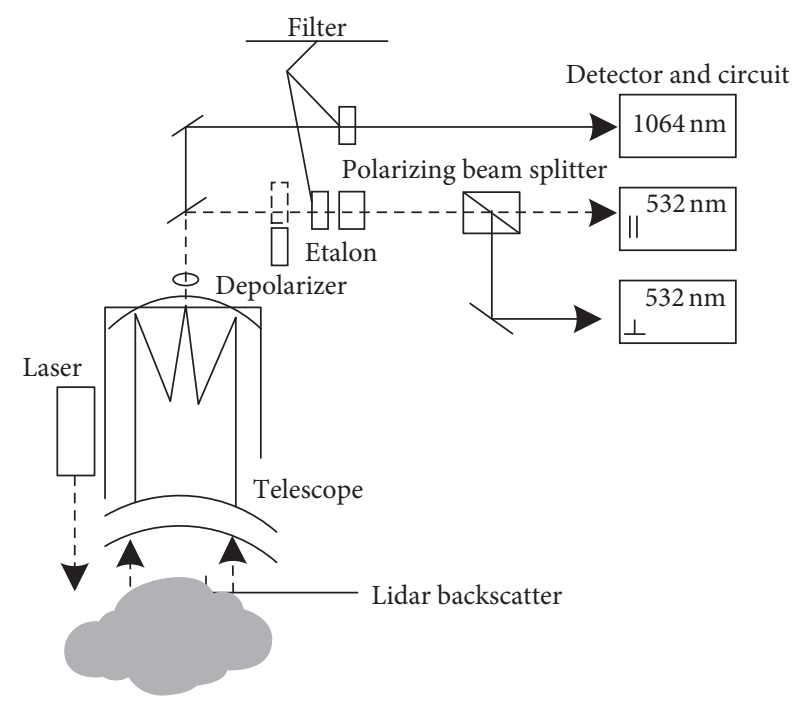

FIGURE 2: Optical path of emission and receipt module in CALIOP.

are the distance from the target to the receiving module, atmospheric laser echo signal, radar calibration parameters, cloud meter backscatter coefficient, molecular Rayleigh backscatter coefficient, cloud meter scattering extinction coefficient, and molecular Rayleigh scattering extinction coefficient.

$$
\begin{aligned}
P(z)= & \frac{C}{z^{2}}\left[\beta_{R}\left(\lambda_{0}, z\right)+\beta_{M}\left(\lambda_{0}, z\right)\right] \exp \\
& \cdot\left\{-2 \int_{0}^{z}\left[\alpha_{R}\left(\lambda_{0}, \xi\right)+\alpha_{M}\left(\lambda_{0}, \xi\right)\right] d \xi\right\} .
\end{aligned}
$$

The total backscattering coefficient $\beta$ is the sum of the cloud rice backscattering coefficient $\beta_{M}$ and the molecular Rayleigh backscattering coefficient $\beta_{R}$, and the total extinction coefficient $\alpha$ is the sum of the cloud rice scattering extinction coefficient $\alpha_{M}$ and the molecular Rayleigh scattering extinction coefficient $\alpha_{R}$. The total backscatter can also be expressed as the sum of the backscatter coefficients of the polarized laser light in the vertical and parallel directions. The degree of backscattering depolarization is defined as the ratio of the backscattering coefficient of polarized laser light in the vertical direction to the backscattering coefficient of polarized laser light in the parallel direction, as shown in the following equation: 


$$
\delta(z)=\frac{\beta_{\perp}(z)}{\beta_{\|}(z)},
$$

where $\delta(z)$ is the degree of depolarization of the target backscatter, $\beta_{\perp}(z)$ is the backscattering coefficient of the polarized laser light in the vertical direction, and $\beta_{\|}(z)$ is the backscattering coefficient of the polarized laser light in the parallel direction. Radar laser scattering calculations are extremely complex. To simplify the calculation process, theoretical inversion calculations can be performed by combining empirical parameters. The commonly used Monte Carlo method can simulate the scattering phenomenon of water vapor cloud and spherical particle aerosol. The backscattering coefficient $A_{s}$ integrated over the optical thickness and the degree of depolarization $\delta$ of the optical thickness are used to approximate the expression of the spherical particle scattering. The expression is as follows:

$$
A_{s}=\frac{\gamma_{s s}}{\gamma^{\prime}}=0.999-3.906 \delta+6.263 \delta^{2}-3.554 \delta^{3},
$$

where $\gamma_{s s}$ is the backscattering coefficient of the primary scattering integrated at the corresponding optical thickness and $\gamma^{\prime}$ is the total decay of the backscattering coefficient of the primary scattering and several scatterings. Equation (3) can be further simplified to

$$
\frac{1}{A_{s}}=\frac{\gamma_{s s}+\gamma_{m s}}{\gamma_{s s}}=\left(\frac{1+\delta}{1-\delta}\right)^{2}
$$

This simplified formula can be applied to the atmospheric echo signals of spherical particles (cloud droplets and liquid aerosol particles), regardless of extinction coefficient or particle size. The relationship calculated using Monte Carlo probability is basically consistent with the data of spherical particles detected by the CALIPSO satellite.

3.2. Simulated Annealing Algorithm. A simulated annealing algorithm is an algorithm for solving large-scale data optimization problems. During the annealing process of the material processing, the temperature of the material gradually decreases, and a quasiequilibrium to equilibrium state change occurs at multiple temperature nodes until its temperature drops to a minimum. The iterative process of the simulated annealing algorithm is to simulate the annealing phenomenon, and on this basis, the locally optimal solution is avoided by continuously perturbing. The specific perturbation method is to make the value jump out of a small local range by selecting some boundary points that may lead to poor results. The calculation of laser scattering has the characteristics of extremely large calculation volume and high calculation accuracy. The simulated annealing algorithm can simplify the calculation under the premise of ensuring the calculation accuracy. Therefore, the simulated annealing algorithm is used to perform the inversion operation of the atmospheric cloud distribution polarization backscatter.

If the internal energy of the $i$ iteration state is $E_{i}$ and the internal energy of the $i+1$ state generated by the disturbance is $E_{i+1}$, then the following formula holds:

$$
p=\exp \left(-\frac{E_{i+1}-E_{i}}{k T}\right)
$$

where $T$ is the $i$ temperature. $k$ is Boltzmann's constant, usually taken as 1 . If the internal energy difference $E_{i+1}-$ $E_{i}=\Delta E$ is a negative number, indicating that the internal energy has decreased, the state at the $i+1$ time is set to the current state. If the internal energy difference is a positive number, it is judged whether the state of the $i+1$ time is set as the current state by generating a random number. The specific way to judge the random number is to randomly generate $q$ in $(0,1)$ and update the status when $q>p$ and, otherwise, refuse to update. Through several times of internal energy difference judgment, random number comparison, and state update, the state can be dynamically balanced and continue to lower the temperature and refind the equilibrium state at the corresponding temperature until the temperature drops to the minimum and approaches zero. At this time, the internal energy of the system has reached the minimum level, and the minimum internal energy is the objective function of the simulated annealing algorithm. The calculation process of the simulated annealing algorithm is shown in Figure 3. When the temperature is high, the algorithm state is more, which is conducive to the search for the most advantages. When the temperature is low, the algorithm state is less, which is conducive to global convergence.

A simulated annealing algorithm does not require additional information in the calculation and can solve nondifferentiable and discontinuous functions. Therefore, simulated annealing algorithm has problems in multiextreme objective function problems, multiextreme nonconvex feasible domain problems, and disconnected feasible domain problems. There are advantages that traditional algorithms do not have in solving complex problems. The essence of the simulated annealing algorithm is that the algorithm has the probability of receiving nonoptimal solutions and introduces temperature variables. The reception of nonoptimal solutions is the biggest feature of the simulated annealing algorithm. The introduction of the annealing mechanism and the perturbation mechanism allows the algorithm to search for both optimized points and degraded points. The generation of each point does not have a definite relationship, so it can help the calculation result jump out of the local optimal value. The optimization process of the algorithm is divided into many different balanced temperature intervals. The introduction of temperature can help the algorithm to choose the degradation point. The decrease in temperature makes the disturbance frequency gradually decrease and helps the algorithm to converge. In addition, the simulated annealing algorithm has some nonspecific advantages, such as no need for auxiliary information, and good at searching complex areas. Compared with other algorithms that also have these advantages, the simulated annealing algorithm is more efficient and requires fewer steps. The main parameters of the simulated annealing algorithm are the initial temperature, the temperature update function, the state generation function, the state reception function, and the number of internal and external cycles. 


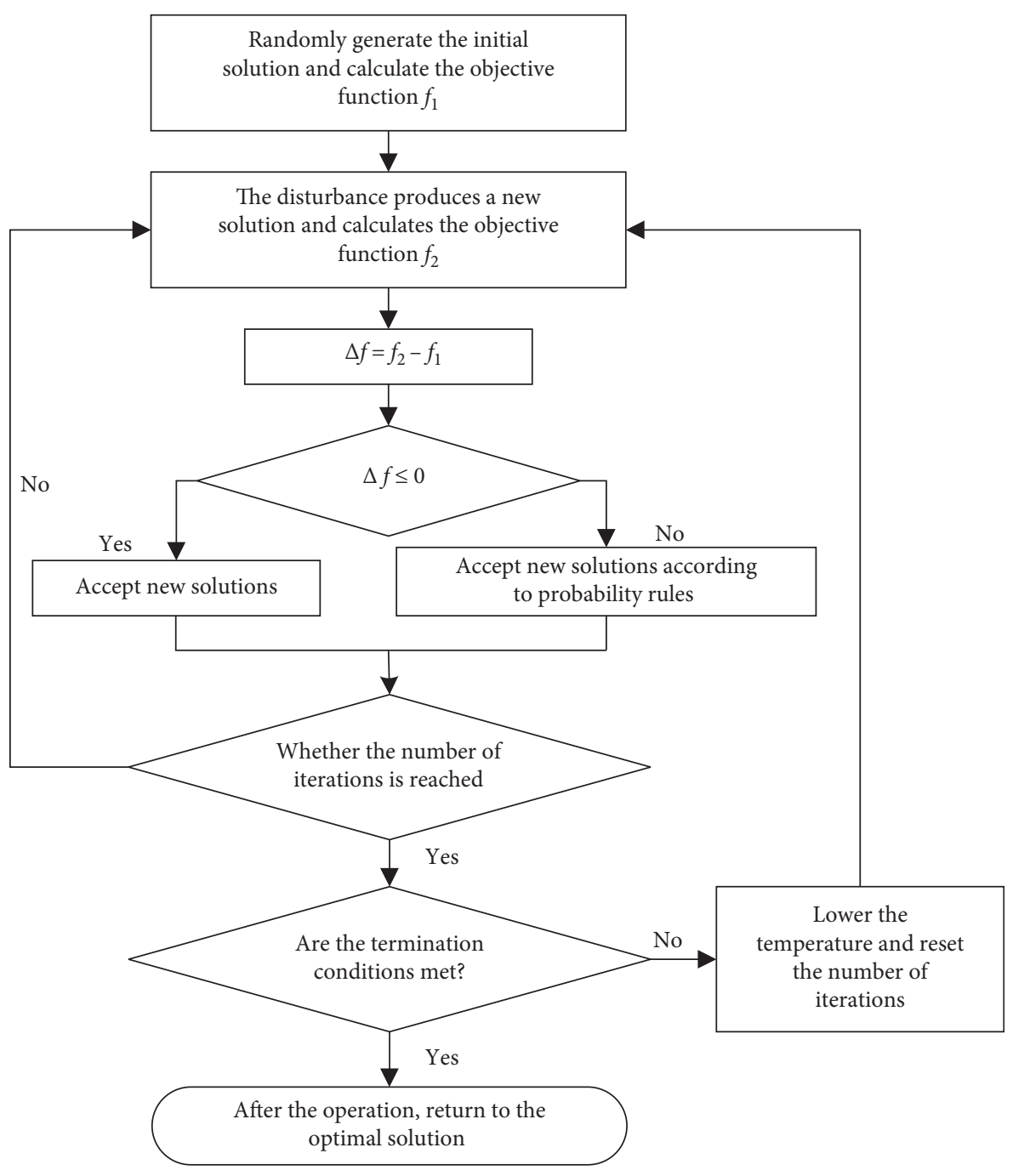

Figure 3: Flowchart of simulated annealing algorithm.

First, we conduct a large area coarse search and then perform a local detailed search. The initial annealing temperature affects the accuracy of the search. A smaller initial temperature corresponds to a smaller search range, and a larger initial temperature corresponds to a larger amount of calculation. Therefore, it is necessary to select a suitable initial temperature value. The slower temperature update function will increase the number of iterations that the algorithm needs to perform, and the faster decay temperature update function accesses the neighborhood and receives less solution. Commonly used temperature update functions include logarithmic decline, rapid cooling, and exponential decline. The state generating function should make the range of candidate solutions as large as possible, while the state receiving function has almost no effect on the performance of the algorithm.

\subsection{Analysis of Atmospheric Cloud Distribution Based on} Simulated Annealing Algorithm. CALIPSO data usually contains two levels. The first-level data used in this study includes the attenuation backscatter coefficient sequence of the $532 \mathrm{~nm}$ vertical radar channel, the $532 \mathrm{~nm}$ parallel radar channel, and the $1064 \mathrm{~nm}$ infrared radar channel. By separating and extracting the above data, the $532 \mathrm{~nm}$ vertical polarization component and the $532 \mathrm{~nm}$ horizontal polarization component can be obtained. Using the simulated annealing algorithm to perform the inversion operation, the backscattering coefficient and depolarization coefficient of the cloud layer at $532 \mathrm{~nm}$ wavelength can be obtained. Therefore, the degree of depolarization of backscattering can be obtained by detecting clouds and aerosols at different heights above a certain ground. Figure 4 is a graph of the depolarization degree and backscattering coefficient of ice crystal cloud and water vapor cloud.

It can be seen from Figure 4 that the two curves meet at one point. The degree of depolarization on the right side of the intersection point means that the ice cloud is composed of randomly oriented ice crystal particles. At this time, the degree of depolarization depends on the nature of the ice crystal particles and is related to the signal strength. In the upper left area of the intersection, the degree of depolarization is much smaller than the backscattering coefficient. This is because the nature of the ice crystal cloud is determined by the ice crystal polymer in the horizontal direction. The specular reflection 


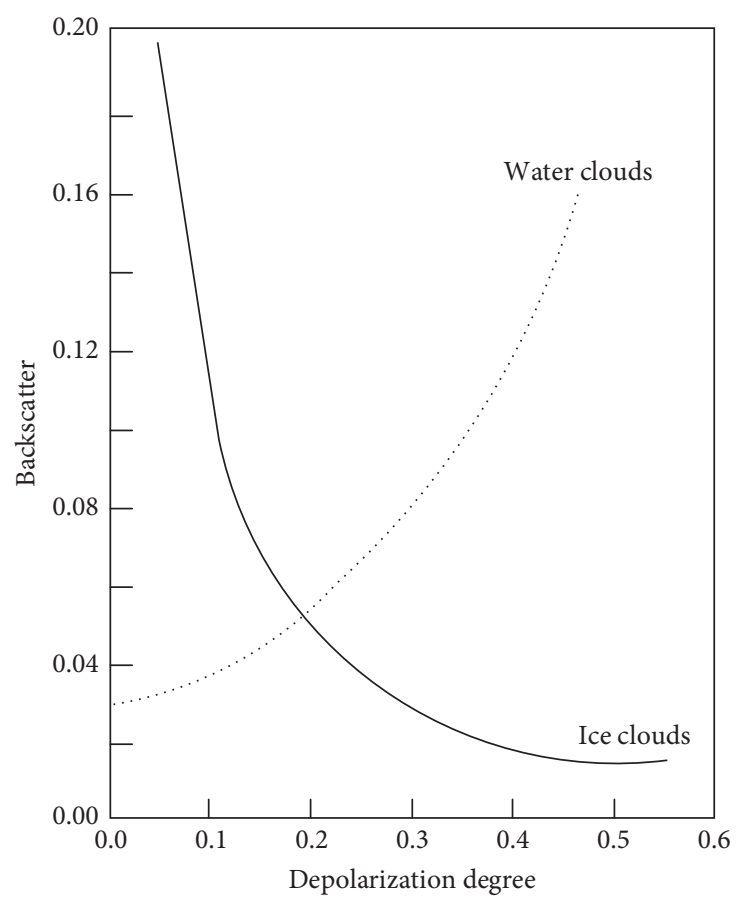

FIgURE 4: The curve between depolarization degree and backscattering coefficient of ice clouds and water clouds.

generated by the horizontal polymer does not affect the backscattering polarization of the incident laser light. At the same time, for the ice crystal polymer in the horizontal direction, the value of the backscattering coefficient will be large. When the proportion of ice crystal aggregates in the horizontal direction decreases, the proportion of other types of ice crystal particles increases, resulting in an increase in the value of depolarization. Therefore, the depolarization degree of the ice crystal cloud is inversely proportional to the backscattering coefficient. For the water vapor cloud, the degree of depolarization is positively correlated with the backscattering coefficient. Therefore, the types of clouds can be judged by studying the degree of depolarization and the backscattering coefficient of atmospheric clouds.

\section{Experimental Design and Analysis}

The CALIOP spaceborne lidar is used to observe the summer and winter atmospheric clouds in a certain place, and the simulated annealing algorithm is used to invert the data obtained by the observation. The initial temperature of the simulated annealing algorithm is set to $T_{0}=1$, the temperature update function parameter $a=0.6$, the state generation function boundary step $s=0.05$, and the search step $c=0.2$. The simulated annealing algorithm is used to obtain the backscatter coefficient and depolarization degree corresponding to different seasons and weather. The experiment found that the detection distance of CALIOP in winter is much lower than that in summer. This is because the intensity of the laser signal of the atmospheric radar in winter is much lower than that in summer, and the laser signal decays rapidly in the clouds. The depolarization degree of clouds and aerosols in winter is slightly greater than that in summer. The backscatter coefficient and the degree of depolarization corresponding to different altitudes in summer sunny weather are shown in Figure 5.

As can be seen from Figure 5, due to the sunny weather, the concentration of aerosol particles in the atmosphere is low. Since the degree of depolarization is low and the change with height is not very significant, it can be considered that there are very few nonspherical particles in the atmosphere, and the possibility of cloud distribution is low. The relationship between the degree of depolarization and the backscattering coefficient with height in cloudy summer weather is shown in Figure 6.

In Figure 6, there are cloud distributions at heights of $4.17 \mathrm{~km}$ and $5.21 \mathrm{~km}$. There is also a large degree of depolarization below the height of $550 \mathrm{~m}$. This is due to the large traffic flow and less vegetation coverage at the height near the ground. These factors lead to the distribution of more dust and other particulate matter in the air below $550 \mathrm{~m}$, which is a nonspherical aerosol. When the height is increased to about $4.17 \mathrm{~km}$, the degree of depolarization and the backscattering coefficient are significantly improved. This is because the nonspherical particles in the atmospheric cloud are relatively sparse, and the relatively dense spherical water droplets are backscattered several times, which improves a certain degree of depolarization. Therefore, it can be judged that the type of cloud layer near the height of $4.17 \mathrm{~km}$ may be a water vapor cloud. When the height is further increased to $5.21 \mathrm{~km}$, the depolarization value decreases significantly, indicating that the cloud layer at this height is extremely thin, and the proportion of nonspherical particles in the cloud layer is very small, so the type of cloud at this location can also be judged as water vapor cloud.

Figure 7 is a graph showing the relationship between the degree of depolarization and the backscattering coefficient at 


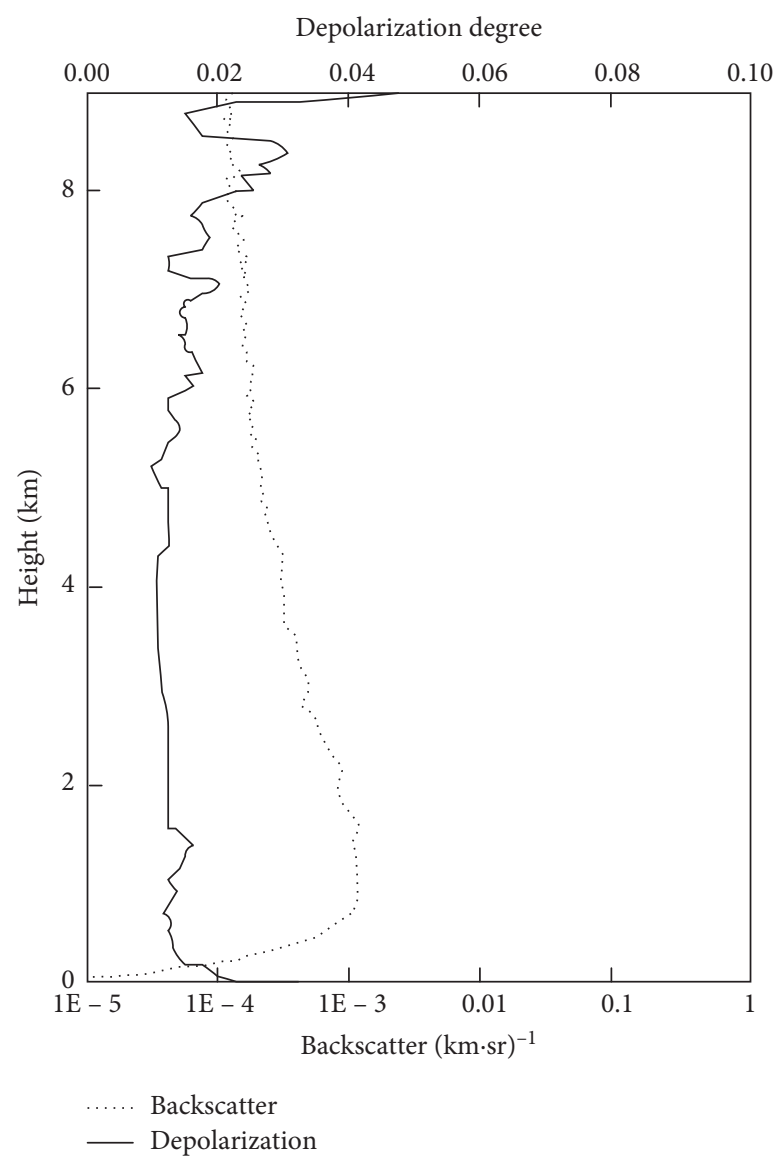

FIgURE 5: Curve between depolarization degree and backscattering coefficient in fine summer days.

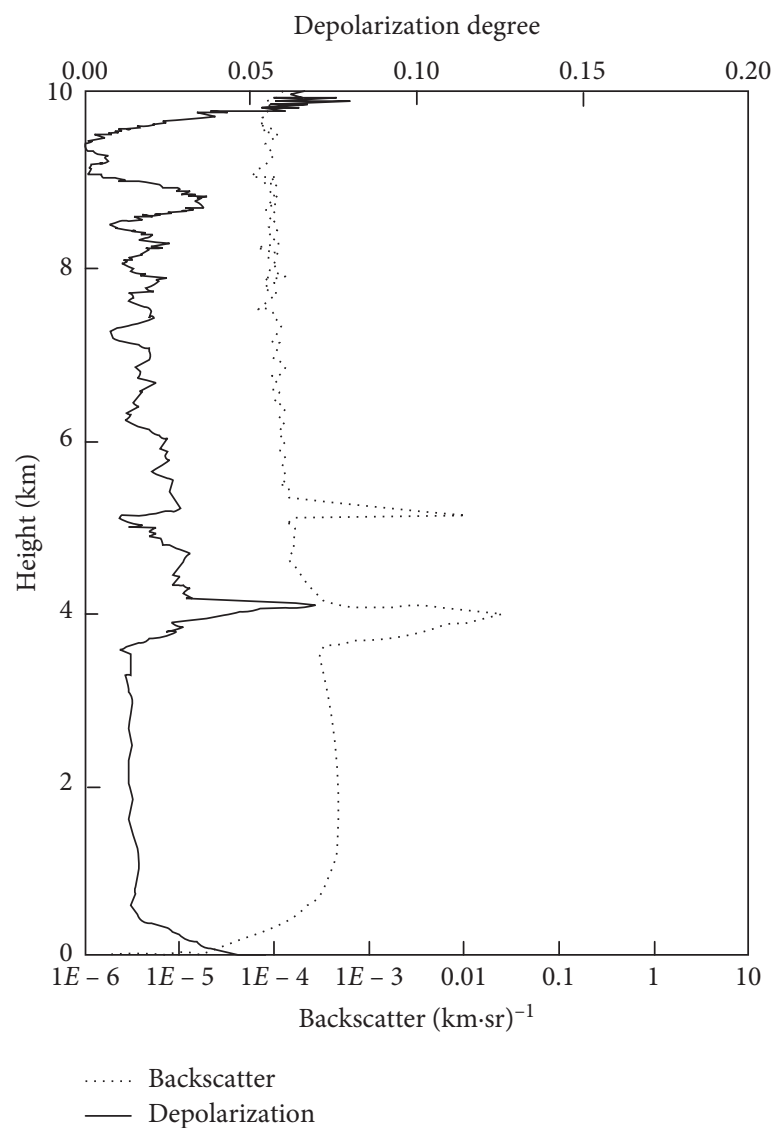

Figure 6: The curve between depolarization degree and backscattering coefficient on a cloudy summer day. 


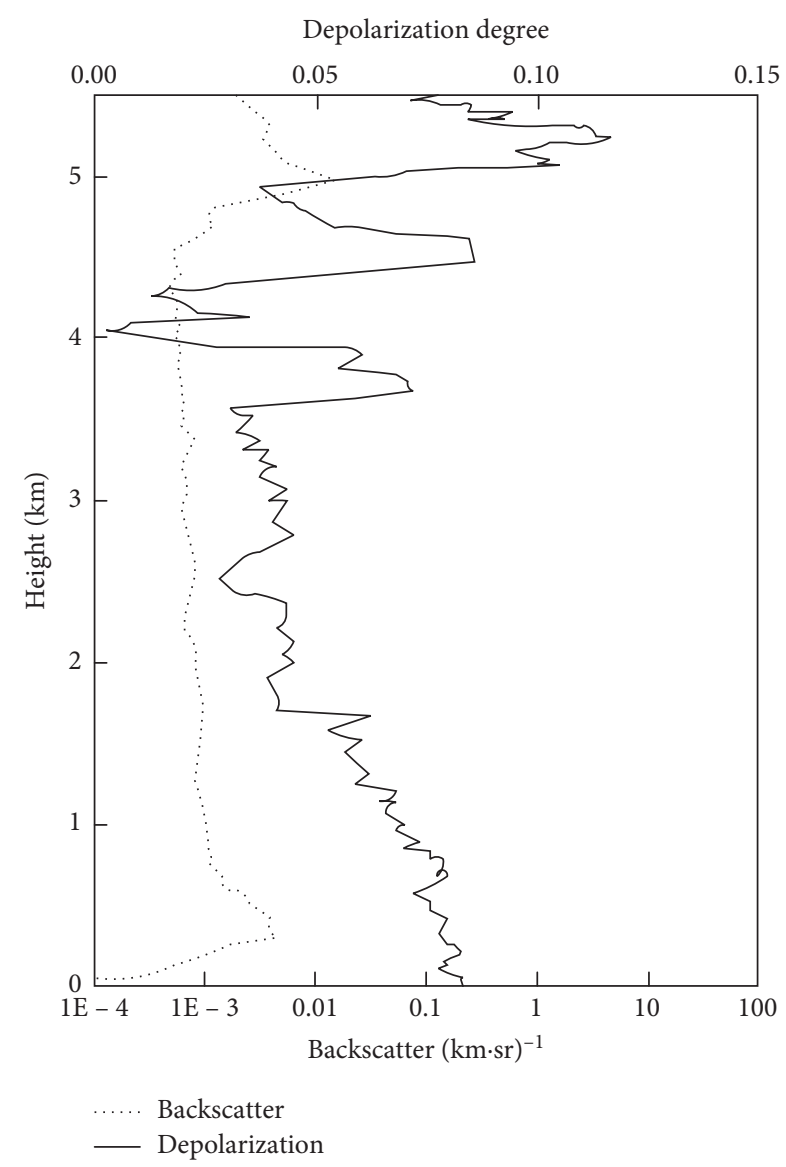

FIgURE 7: The curve between depolarization degree and backscattering coefficient on a cloudy winter day.

different heights in cloudy winter weather. At the height of $5.26 \mathrm{~km}$, both the degree of depolarization and the backscattering coefficient increase significantly, and it can be judged that there is cloud distribution at this height. Because the relationship between the degree of depolarization and the backscattering coefficient increases at the same time, that is, there is a proportional relationship, indicating that the cloud layer contains fewer ice crystal particles, so it can be judged that the cloud layer at this height is a water vapor cloud. The backscattering of spherical water droplets in the water vapor cloud causes the depolarization degree and backscattering coefficient of this type of cloud to rise simultaneously.

Figure 8 is a distribution diagram of depolarization degree and backscattering coefficient at different levels in the winter atmosphere with severe haze. At a height of $0.49 \mathrm{~km}$, the backscattering coefficient increased significantly, while the degree of depolarization decreased slightly, indicating that the height may be distributed with a small amount of horizontal ice crystal particles or a large number of aerosols caused by atmospheric pollution and heavy haze caused by the distribution of nonspherical particles. At a height above $1 \mathrm{~km}$, there is a relatively small degree of depolarization, because the distribution of pollutant particles present at a high altitude is significantly lower than at a lower altitude.

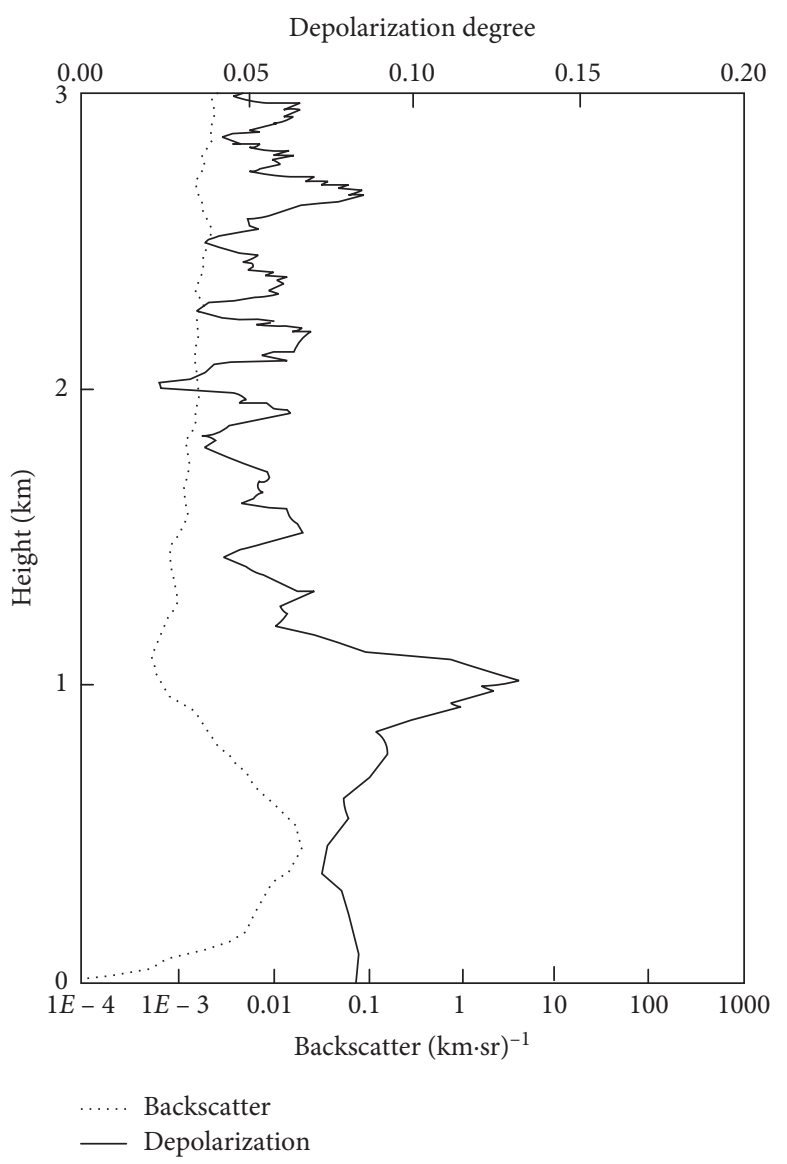

FIGURE 8: The curve between depolarization degree and backscattering coefficient on a hazy winter day.

\section{Conclusion}

The launch of meteorological satellites allows the optical properties of atmospheric clouds and aerosols to be more accurately measured, thereby allowing meteorological changes to be more accurately predicted. In order to study the backscattering of polarized laser light distributed in atmospheric clouds, this paper uses a simulated annealing algorithm to analyze the data of spaceborne lidar and obtains the backscattering coefficient and depolarization degree of atmospheric clouds and aerosols at $532 \mathrm{~nm}$ wavelength. In order to verify the inversion performance of the simulated annealing algorithm, the simulated annealing algorithm is applied to the actual observation data of the spaceborne lidar. According to the simulated annealing algorithm inversion, the backscattering coefficient and depolarization degree of atmospheric clouds and aerosols corresponding to different altitudes under cloudy, sunny, haze, and other weather conditions in summer and winter, and the types and states of clouds and aerosols are analyzed. In the example analysis, there are very few nonspherical particles in the atmosphere of a certain place in summer in sunny weather, and there is almost no cloud layer distribution at this time. In cloudy summer weather, there is a cloud of water vapor distribution above $4 \mathrm{~km}$, and there are a large number of nonspherical soot particles below $550 \mathrm{~m}$. Under cloudy 
weather in winter, there is a distribution of water vapor clouds above $5 \mathrm{~km}$. In winter fog and haze, there are a large number of nonspherical particles or horizontal ice crystal particles caused by pollution in the atmosphere near $500 \mathrm{~m}$. The distribution of atmospheric haze particles above $1 \mathrm{~km}$ has dropped sharply. The result of the example proves that the spaceborne polarized lidar using the simulated annealing algorithm for data inversion can detect the backscattering of atmospheric clouds and aerosols under different weather conditions. The simulated annealing algorithm has a strong performance as a radar data inversion algorithm. This study has some shortcomings, that is, the degree of depolarization and backscattering coefficient are not used to further derive the weather forecast. In future work, we can improve the algorithm by introducing the correlation analysis between the weather forecast and cloud type, so that the spaceborne lidar data can directly generate the weather forecast.

\section{Data Availability}

The data used to support the results of this study are included in the references.

\section{Conflicts of Interest}

The authors declare that they have no conflicts of interest.

\section{Acknowledgments}

This work was supported by the Shaanxi Provincial Department of Education Special Scientific Research Project (18JK0831), the Key Research and Development Plan Project of Xianyang City (2019K02-21), and Xianyang Normal University "Youth Key Teacher" Training Project (XSYGG201803).

\section{References}

[1] M. G. Giacomo, R. Marc, L. Xavier et al., "Seafloor change detection using multibeam echosounder backscatter: case study on the Belgian part of the North Sea," Marine Geophysical Researches, vol. 39, pp. 229-247, 2018.

[2] P. Ponomarenko, J. P. St-Maurice, and K. A. Mcwilliams, "Calibrating HF radar elevation angle measurements using E layer backscatter echoes," Radio Science, vol. 53, no. 11-12, pp. 1438-1449, 2018.

[3] S. P. Rusli, D. P. Donovan, and H. W. J. Russchenberg, "Simultaneous and synergistic profiling of cloud and drizzle properties using ground-based observations," Atmospheric Measurement Techniques, vol. 10, no. 12, pp. 4777-4803, 2017.

[4] Z. Tian, Y. Tian, B. Wen et al., "Wave-height mapping from second-order harmonic peaks of wide-beam HF radar backscatter spectra," IEEE Transactions on Geoscience and Remote Sensing, vol. 58, no. 2, pp. 925-937, 2020.

[5] G. Wang, F. Gao, R. Fan, and C. Tellambura, "Ambient backscatter communication systems: detection and performance analysis," IEEE Transactions on Communications, vol. 64, no. 11, pp. 4836-4846, 2016.

[6] D. Wallis, L. N. Hansen, T. Ben Britton, and A. J. Wilkinson, "Geometrically necessary dislocation densities in olivine obtained using high-angular resolution electron backscatter diffraction," Ultramicroscopy, vol. 168, pp. 34-45, 2016.
[7] T. Nozue, K. Fukui, Y. Koyama et al., "Effects of sitagliptin on coronary atherosclerosis evaluated using integrated backscatter intravascular ultrasound in patients with type 2 diabetes: rationale and design of the TRUST study," Heart and Vessels, vol. 31, no. 5, pp. 649-654, 2016.

[8] B. Lyu, H. Guo, Z. Yang, and G. Gui, "Throughput maximization for hybrid backscatter assisted cognitive wireless powered radio networks," IEEE Internet of Things Journal, vol. 5, no. 3, pp. 1-7, 2018.

[9] X. Lurton, D. Eleftherakis, and J.-M. Augustin, "Analysis of seafloor backscatter strength dependence on the survey azimuth using multibeam echosounder data," Marine Geophysical Research, vol. 39, no. 1-2, pp. 183-203, 2017.

[10] M. Haeffelin, Q. Laffineur, J.-A. Bravo-Aranda et al., "Radiation fog formation alerts using attenuated backscatter power from automatic lidars and ceilometers," Atmospheric Measurement Techniques, vol. 9, no. 11, pp. 5347-5365, 2016.

[11] A. Gebru, M. Brydegaard, E. Rohwer et al., "Probing insect backscatter cross section and melanization using $\mathrm{kHz}$ optical remote detection system," Journal of Applied Remote Sensing, vol. 11, no. 1, pp. 1-12, 2017.

[12] S. H. Kim and D. I. Kim, "Hybrid backscatter communication for wireless-powered heterogeneous networks," IEEE Transactions on Wireless Communications, vol. 16, no. 10, pp. 6557-6570, 2017.

[13] R. D. Lindsley and D. G. Long, "Enhanced-resolution reconstruction of ASCAT backscatter measurements," IEEE Transactions on Geoscience and Remote Sensing, vol. 54, no. 5, pp. 2589-2601, 2016.

[14] J. Qian, F. Gao, G. Wang, S. Jin, and H. Zhu, "Noncoherent detections for ambient backscatter system," IEEE Transactions on Wireless Communications, vol. 16, no. 3, pp. 1412-1422, 2017.

[15] Z. Wenwen, Y. Fulian, C. Juanfang et al., "The effect of send/ receive dual channel parameters on polarization parameters measurement," Chinese Journal of Electronics, vol. 26, no. 2, pp. 118-126, 2017. 